(CASE REPORT)

\title{
Lung contusion mimicking COVID-19 pneumonia: A case report
}

\author{
Erenler AK*, Korkmaz Ü and Ergün EC \\ Affiliation for all authors: Hitit University, School of Medicine, Department of Emergency Medicine, Çorum, Turkey.
}

Publication history: Received on 21 April 2020; revised on 04 May 2020; accepted on 09 May 2020

Article DOI: https://doi.org/10.30574/wjarr.2020.6.2.0115

\begin{abstract}
In December 2019, a series of pneumonia cases of unknown origin were determined in Wuhan, China. The disease, then, was named as 2019 novel coronavirus (2019-nCoV) and subsequently as COVID-19 officially by the World Health Organization (WHO). Its characteristics such as high infectivity and mortality makes COVID-19 a serious public health problem. It causes consumption of sources in healthcare facilities dealing with the disease. In this case report, we present you a patient admitted to our Emergency Department due to multiple trauma and whose computed tomography findings were confused with COVID-19 pneumonia
\end{abstract}

Keywords: COVID-19; trauma; lung contusion; pneumonia

\section{Introduction}

In December 2019, a series of pneumonia cases of unknown origin were determined in Wuhan, China. Lately, the disease was diagnosed as being caused by a novel coronavirus named initially as 2019 novel coronavirus (2019-nCoV) and subsequently as COVID-19 officially by the World Health Organization (WHO) on February 11, 2020 [1]. The first confirmed case of COVID-19 in Turkey was recorded in March 10 th 2020 . Since then, 56956 confirmed cases and 1198 deaths were announced by Turkish Ministry of Health [2]. Coronaviruses belong to the family Coronaviridae and the order Nidovarales, a family that includes viruses that cause diseases ranging from the common cold to severe acute respiratory syndrome (SARS) and the Middle East respiratory syndrome (MERS) [3]. Its characteristics such as high infectivity and mortality makes COVID-19 a serious public health problem [4]. The main symptoms of the disease are fever, dry cough, and fatigue [5]. The gold standard for diagnosis of 2019-nCoV infection is detection of the new coronavirus nucleic acid in swabs, sputum, secretions from the lower respiratory tract, or blood [6]. Although, the definitive diagnosis of COVID-19 infection is based on reverse transcription polymerase chain reaction (PCR), radiological findings are important for the diagnosis of pneumonia. Timely diagnosis of COVID-19 pneumonia would enable rapid management planning including further imaging follow-ups and conservative care. Given the fact that the most frequent imaging finding is ground-glass opacity (GGO), careful interpretation of the chest radiographs is needed as GGOs may look very faint. CT scans are recommended in patients with suspicious lung abnormality [7]. However, there are also some challenges in CT diagnosis of the disease. The radiological manifestations of the disease may be misdiagnosed or confused with findings of other entities. In this report, we present you a case of a male admitted to our Emergency Department (ED) due to multiple trauma. It was a challenge to determine whether the reason for GGO was lung contusion or COVID-19.

\section{Case Presentation}

A 46-year-old male patient presented to our ED due to trauma following motor vehicle accident. On admission, his vital signs were normal and he was conscious. The patient was physically healthy before and had no underlying diseases. On physical examination, he had tenderness on his right arm and right leg. His blood samples were taken and patient was transferred to radiology room for X-Ray and CT examinations. His complete blood count and biochemistry analyses

\footnotetext{
${ }^{*}$ Corresponding author: Erenler AK
}

Copyright (C) 2020 Author(s) retain the copyright of this article. This article is published under the terms of the Creative Commons Attribution Liscense 4.0. 
were as follows: Hemoglobin: $13.2 \mathrm{~g} / \mathrm{dL}$, blood urea nitrogen (BUN): $26 \mathrm{mg} / \mathrm{g} / \mathrm{dL}$, Aspartate Aminotransferase (AST): 70 U/L, Alanine Aminotransferase (ALT): 74 U/L, Lactic Acid Dehydrogenase (LDH): 393 U/L, Creatine Kinase (CK): $1604 \mathrm{U} /$ L. On X-Ray, a fracture in humeral shaft fracture and intertrochanteric femur fracture were determined (Figure 1 and 2). On thorax CT; bilateral, multiple GGOs were determined. (Figure 3). Even by radiologists, a definite differential diagnosis of COVID-19 pneumonia and lung contusion could not be made. After initial interventions in the ED, the patient was transferred to an operation room designed for COVID-19 patients. All the surgeons and nurses involved in the operation wore protective clothes. The day after, PCR result of the patient for COVID-19 was reported as negative.

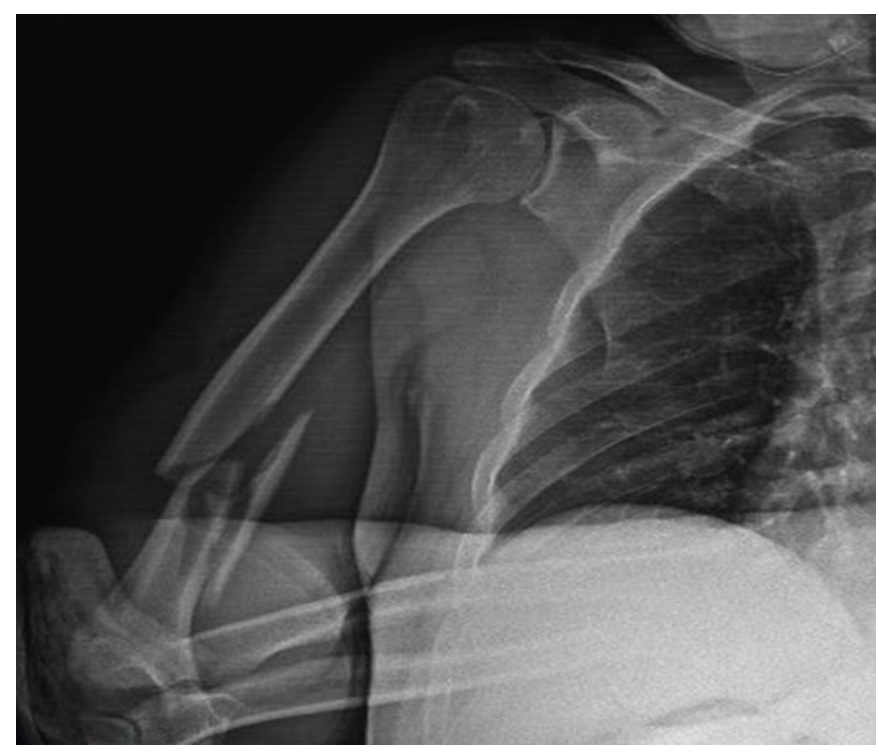

Figure 1 Humerus shaft fracture of the patient

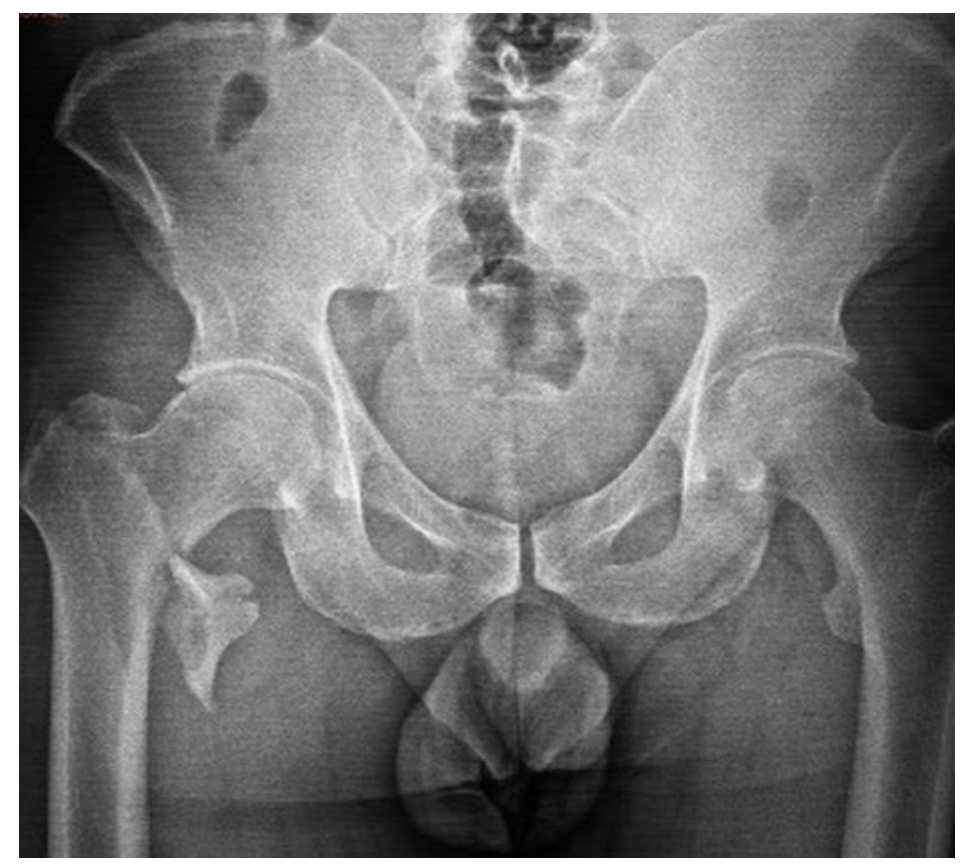

Figure 2 Interthrocanteric femur fracture of the patient 


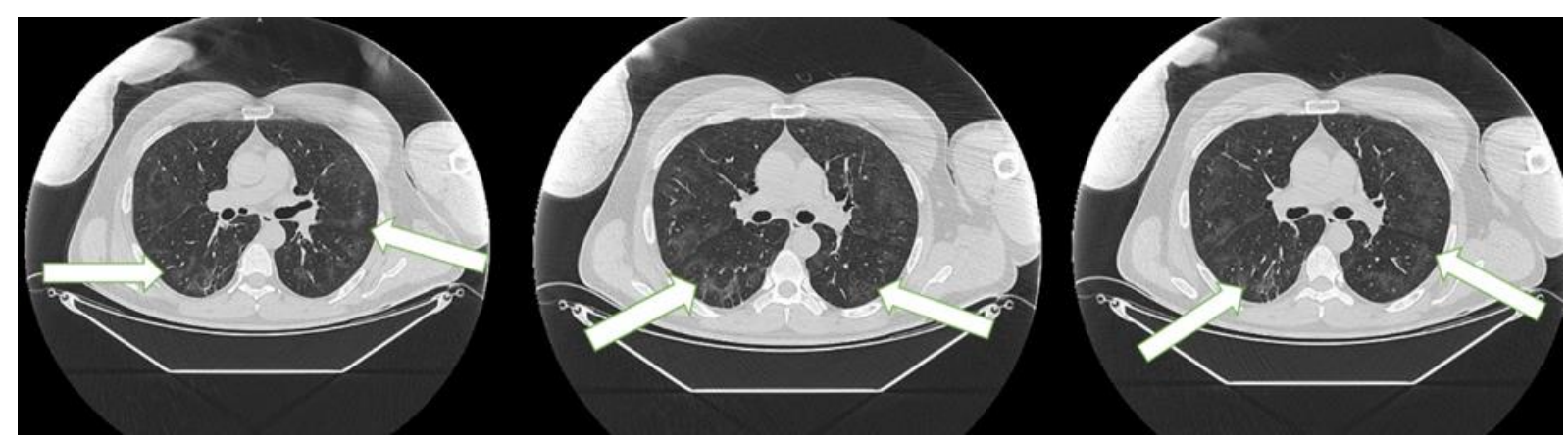

Figure 3 White arrows indicate the sites of lung contusion mimicking COVID-19 pneumonia

\section{Discussion}

COVID-19 creates risk not for only physicians fighting in the front line but also for those who have to deal with various diseases, including trauma. CT findings of typical COVID-19 cases are known to be single or multiple patchy GGOs accompanied by septal thickening [8]. CT imaging can play an important role in the early diagnosis and disease stratification of COVID-19. The size and type of CT abnormalities are related to disease severity [9]. The differential diagnosis of lung contusion from other pulmonary diseases, or pneumonia may be problematic [10]. In our case, CT findings of the patient were confused with COVID-19 and even the radiologists failed to make a definite diagnosis. In lung contusion, alveoli and capillaries are torn, blood and interstitial fluid leak into the alveoli and tissues. First, a haematoma appears in the area of the injury, then, several hours later, oedema develops in and around the affected area [11]. Besides, in COVID-19, the main features are GGOs suggesting inflammation of lung parenchyma mainly located in the peripheral zones of both lungs [12]. Similarities in CT images of both diseases may cause challenges in differential diagnosis.

\section{Conclusion}

COVID-19 pneumonia is a diagnostic challenge not only for physicians in the front-line in the warfare against the disease (ED physicians, infection diseases specialists, pulmonologists, etc), but also for other specialists who have to manage this disease, indirectly. COVID-19 pneumonia may mimic numerous diseases including trauma. In conclusion, COVID19 may cause consumption of human and economic sources. Physicians must be aware of other conditions that may be confused with COVID-19.

\section{Compliance with ethical standards}

\section{Acknowledgments}

None to declare

\section{Disclosure of conflict of interest}

Authors declare no conflict of interests

\section{Statement of ethical approval}

The present research work does not contain any studies performed on animals/humans subjects by any of the authors.

\section{Statement of informed consent}

The study does not involve information about any individual 


\section{References}

[1] Center for Disease Control and Prevention (CDC). Locations with confirmed COVID-19 cases. Available at: https://www.cdc.gov/coronavirus/2019-ncov/locations-confirmed-cases.html, Accessed date: 15 February 2020.

[2] Turkish Ministry of Health Official Website. Available at: https://covid19bilgi.saglik.gov.tr/tr/gunluk-vaka.html

[3] Novel Coronavirus (2019-nCoV). World Health Organization. https://www. who.int/emergencies/diseases/novel-Coronavirus-2019. Published January 7, 2020.

[4] Yang X, Yu Y, Xu J, et al. (2010). Clinical course and outcomes of critically ill patients with SARS-CoV-2 pneumonia in Wuhan, China: a single-centered, retrospective, observational study. Lancet Respir Med.

[5] Huang C, Wang Y, Li X, et al. (2020). Clinical features of patients infected with 2019 novel coronavirus in Wuhan, China. Lancet, 395, 497-506.

[6] Fang X, Zhao M, Li S, Yang L and Wu B. (2020). Changes of CT findings in a 2019 novel coronavirus (2019nCoV) pneumonia patient. QJM.113(4), 271-272.

[7] Kim H. (2020). Outbreak of novel coronavirus (COVID-19): What is the role of radiologists? Eur Radiol.

[8] Chen L, Liu HG, Liu W et al. (2020). Analysis of clinical features of 29 patients with 2019 novel coronavirus pneumonia. Zhonghua Jie He He Hu Xi Za Zhi. 43(3), 203-208.

[9] Liu KC, $\mathrm{Xu} \quad \mathrm{P}, \mathrm{Lv} \quad \mathrm{WF}$, et al. (2020). CT manifestations of coronavirus disease-2019: A retrospective analysis of 73 cases by disease severity. Eur J Radiol. 126,108941.

[10] Rendeki S and Molnár TF. (2019). Pulmonary contusion. J Thorac Dis. 11(Suppl 2), S141-S151.

[11] Cohn SM. (1997). Pulmonary contusion: Review of the clinical entity, J Trauma, 42, 973-9.

[12] An P, Song P, Lian K and Wang Y. (2020). CT Manifestations of Novel Coronavirus Pneumonia: A Case Report. Balkan Med J.

\section{How to cite this article}

Erenler AK, Korkmaz Ü and Ergün EC. (2020 Lung contusion mimicking COVID-19 pneumonia: A case report. World Journal of Advanced Research and Reviews, 6(2), 68-71. 